\title{
Celeganser: Automated Analysis of Nematode Morphology and Age
}

\author{
Linfeng Wang $^{\dagger}$, Shu Kong*, Zachary Pincus ${ }^{\ddagger}$, Charless Fowlkes ${ }^{\dagger}$ \\ \{linfenw2, fowlkes\}@ics.uci.edu shuk@andrew.cmu.edu zpincus@wustl.edu \\ ${ }^{\dagger}$ University of California, Irvine $\quad{ }^{*}$ Carnegie Mellon University ${ }^{\dagger}$ Washington University in St. Louis
}

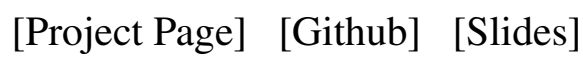

\begin{abstract}
The nematode Caenorhabditis elegans (C. elegans) serves as an important model organism in a wide variety of biological studies. In this paper we introduce a pipeline for automated analysis of C. elegans imagery for the purpose of studying life-span, health-span and the underlying genetic determinants of aging. Our system detects and segments the worm, and predicts body coordinates at each pixel location inside the worm. These coordinates provide dense correspondence across individual animals to allow for meaningful comparative analysis. We show that a model pretrained to perform body-coordinate regression extracts rich features that can be used to predict the age of individual worms with high accuracy. This lays the ground for future research in quantifying the relation between organs' physiologic and biochemical state, and individual life/healthspan.
\end{abstract}

\section{Introduction}

C. elegans is one of the most important invertebrate model organisms in biological research. Landmark studies with C. elegans span various disciplines and fields, including large scale functional characterization of genes [4], complete tracing of cell lineage in developmental [33], and mapping whole-animal nervous system connectomes [6]. C. elegans also offer an ideal model for understanding variability across individuals that result in different health and life span [29, 28]. Even C. elegans, with their famously invariant pattern of embryonic development, experience large differences in individual lifespans. Raised in identical lab environments, genetically identical C. elegans show as much relative variability around their two-week lifespans as humans do around theirs of eighty years [29]. We hope to learn why this is, what more-robust individuals have that their frailer siblings lack, and what that means for human health and aging [29].
In this paper we describe a prototype pipeline for performing high-throughput longitudinal analysis of individual worms. This automated "C. elegans analyzer", which we call Celeganser consists of three computer vision modules: worm segmentation which segments the worm from the plate background,worm body coordinate regression that regress pixels on the worm body to a pre-defined coordinate system, and age regression that predicts the worm's age based on the segmented worm body. The problem of predicting age is of biological interest in its own right as the "physiological age" of an animal does not always match the chronological age. We further hypothesize that if an automated system (e.g., a machine learning model) can regress the body coordinate and age well, it must learn to extract image features that relate to the internal structure of the worm the worm, e.g., organ placement and status (e.g., disorder) that correlates with lifespan and healthspan.

In this paper, we describe the technical aspects of our prototype system and conduct a thorough experimental study to justify the design choices. To summarize our contribution:

1. We propose Celeganser, an automated system assisting analysis of C. elegans for studying lifespan and healthspan and validate the components of the system including segmentation, body-coordinate regression and age regression.

2. We demonstrate that models pre-trained for bodycoordinate regression extract features which are useful in predicting age.

3. We carry out experiments to analyze the extent to which the worm shape and size, internal appearance and background environment are predictive of age. This provides a foundation for future research in lifespan and healthspan.

We start with related work in Section 2, and elaborate on details of our analysis modules in Section 3 . In Section 4 we 
describe a thorough experimental validation, and conclude in Section 5 .

\section{Related Work}

\subsection{Automated analysis of $\mathrm{C}$. elegans}

C. elegans is a microscopic nematode that is used in a wide variety of biological studies and provides one of the best model systems to study lifespan development [16, 18, 12, 17] as their genetics and development is well characterized and the small size of C. elegans makes it possible to grow them in large numbers. Acquiring high-resolution survival data has lead to fruitful discoveries $[8,34,25,15,3]$.

However, this also brings a challenge as manual observation can be tedious and time-consuming. To fully realize the potential for high-throughput studies with strong statistical power, attention has turned to building automated systems that acquire and analyze the worms through automatic microscopic scanning [32, 28]. For example, the lifespan machine is an automated system for imaging large populations of worms using inexpensive hardware (flatbed scanners) to allow for quantitative investigations into the statistical structure of aging as a population [32]. In this paper, we focus on analyzing individual worm at higher resolution (5-10x magnification) in order to to precisely segment the worm from the scanned image, regress body coordinate and estimate its age. Our system is standalone and re-trainable, and produces rich outputs that are useful for a range of downstream studies of individual differences in lifespan and the rate of aging.

We note that a recently published paper [23] carried out a related study, training Convolutional Neural Networks $(\mathrm{CNN})$ for age estimation with worm images as input. Our approach differs in that we image worms automatically (rather than removing individuals and anesthetizing for imaging) allowing us to train and evaluate on a dataset which is 10x larger. We also report age predictions that are somewhat more accurate and can naturally handle a wider variety of poses.

\subsection{Convolutional Neural Networks}

Convolutional Neural Networks (CNN) have become one of the most successful models in machine learning and computer vision for various applications (see e.g., [11, 7, 31]), owing to their flexibility and state-of-the-art performance on many vision tasks [22, 21, 13] especially when leveraging large-scale data (like ImageNet [9]). CNN architectures designed for whole image classification typical involve multiple layers of spatial pooling and produce a single output. For the purpose a producing dense, perpixel predictions and maintaining high spatial resolution, we adopt the U-shaped architecture of [30]. The U-shape architecture include an encoder which is essentially a tra-

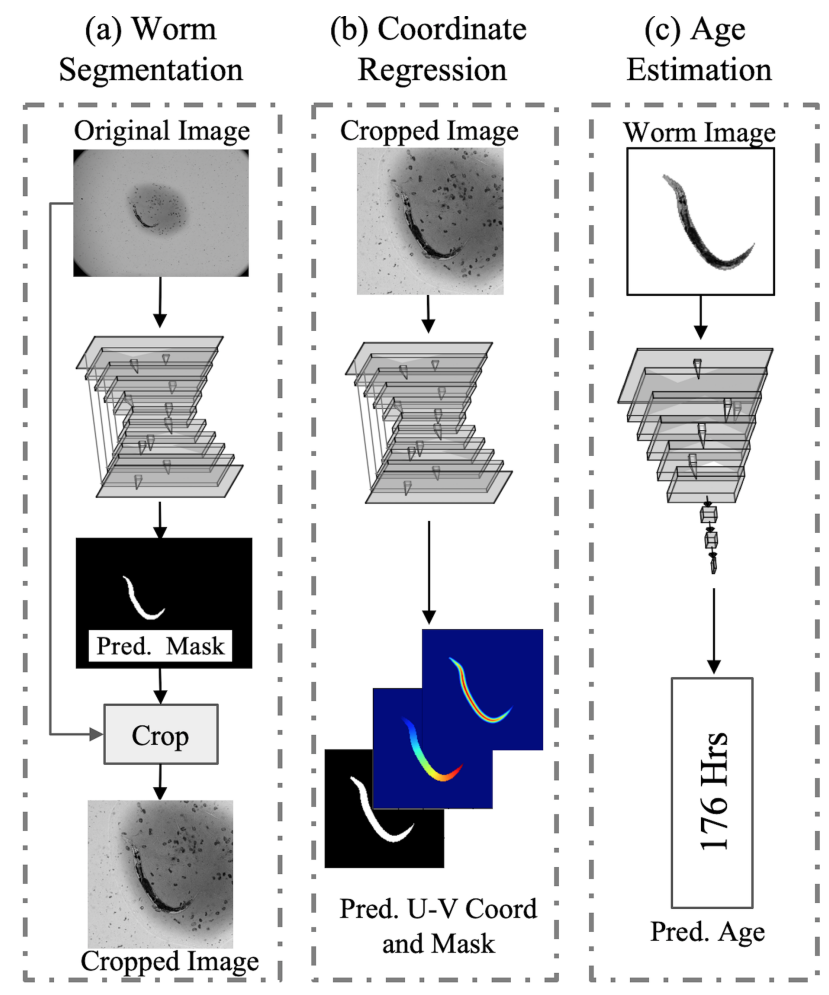

Figure 1: "Celeganser" includes three models: (a) worm segmentation at coarse scale, (b) worm body coordinate regression and (c) age estimation. Using a downsized input image, model (a) predicts a binary segmentation of the worm region. This is used to localize and crop the worm region from the original full-resolution data. The cropped image is then fed to the model (b) for boy coordinate regression and fine segmentation. The segmented worm is the input to the third model (c) for age estimation.

ditional CNN (e.g., ResNet [13]), and a decoder which has skip connections to the encoder layers and upsampling layers.

In addition to outputting discrete per-pixel class labels (which we use for worm segmentation), CNNs can be also trained to output continuous values. Per-pixel regression has been used for tasks such as predicting depth and surface normals [10, 20]. Our approach to mapping worm body coordinates was inspired by the work of Güler et. al. on "DensePose" which estimated dense correspondences between image pixels and surface patches of a canonical 3D human body model [2, 1].

\section{Celeganser: C. elegans Analyzer}

Our system consists of three independently trained models for worm segmentation, worm body coordinate regression and worm age estimation, respectively. Figure 1 shows the flowcharts of the three modules, respectively. Note that 
we train them sequentially, and they also work in a sequential order in the system, as the output of the previous module is the input to the current one. In this section, we elaborate the three models in the same order.

\subsection{Worm Coarse Segmentation and Localization}

In our work, the microscope produces high-resolution images $(2160 \times 2560)$ of individual worms captured once every $\sim 3.5$ hours over the two-week lifespan of the worm. Feeding such a high-resolution image into the CNN module directly for any final predictions is unnecessarily costly in memory and computation. Therefore, we adopt a coarseto-fine strategy. To localize the worm we take as input a downsized image (bilinear interpolated into $512 \times 512$ ) and perform coarse segmentation. The output is a binary mask (after thresholding) of the same size $512 \times 512$. Based on the binary output mask, we crop the raw image for the worm at original scale, and obtain a $960 \times 960$ sub-image capturing the worm. For our data imaged at 5-10x magnification, we found that a $960 \times 960$ sub-image is sufficient to encapsulate any single worm. The cropped sub-image serves as input to the second module for fine-grained prediction.

Predicting a segmentation for the worm can be treated as a two-class semantic segmentation problem (worm foreground vs. background). Using CNNs for semantic segmentation is an active area of research, and many excellent approaches are available in literature [24, 5, 30]. We adopt the U-Net architecture [30], consisting an encoder based on ResNet34 [13] as the backbone, and a decoder which has skip connections to the encoder and upsampling layers for the single mask output at the input resolution. Figure 1 (a) depicts the flowchart of this model.

For training this model, we use a per-pixel binary cross entropy loss. We also insert the loss at multiple scales $(S=$ 5 scales in total) at each skip connection layer, weighted by the resolution of feature activations. The loss for a single image is the sum of binary cross entropy over all scales:

$$
L_{\text {seg }}=-\sum_{s=1}^{S} \frac{1}{\left|\mathcal{I}_{s}\right|} \sum_{i \in \mathcal{I}_{s}}\left(y_{i} \log x_{i}+\left(1-y_{i}\right) \log \left(1-x_{i}\right)\right)
$$

where $x_{i}$ is the predicted probability for pixel $i$ being worm and $\mathcal{I}_{s}$ is the set of pixel predictions at scale $s . y_{i}$ is the ground-truth label, indicating whether the pixel $i$ belongs to worm foreground $\left(y_{i}=1\right)$ or background $\left(y_{i}=0\right)$.

\subsection{Body Coordinate Regression}

The second model, as shown by Figure 1 (b), takes as input the previous cropped sub-image and outputs a fine segmentation mask and worm body coordinate prediction. As we mentioned earlier, our hypothesis with this model is that, once it can regress the worm body well, it should also capture meaningful information from internal structure of

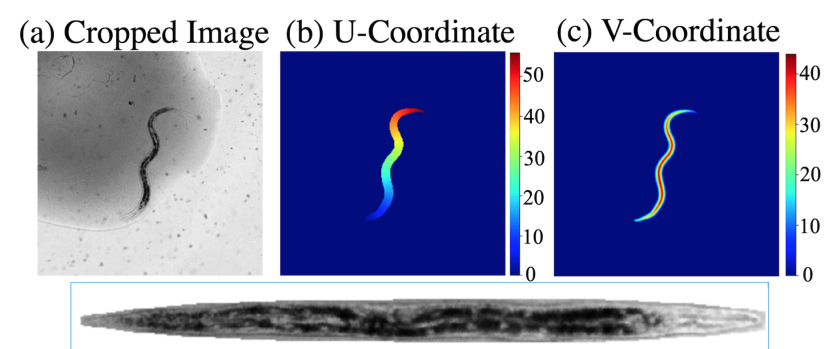

(d) Straightened Worm

Figure 2: For a given cropped image (a), we train for regressing towards its worm UV-coordinate on the body pixels, as shown by (b) and (c) respectively. Based on the predicted coordinates, we can straighten the worm according to a defined "canonical" shape, as shown in (d). This helps us analyze worm age and thus the life/health-span in later work. More straightened worms can be found in Figure 5 with both ground-truth and predicted UV-coordinate.
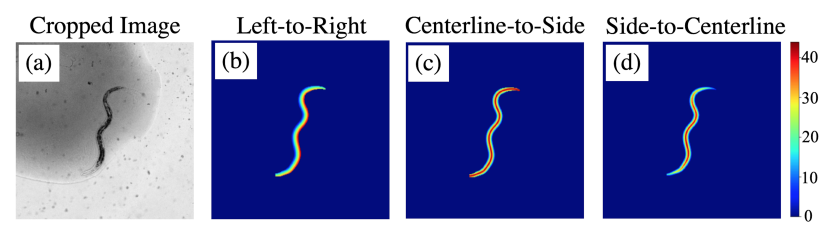

Figure 3: We consider three different representation of the V-coordinate for a given image (a): (b) left-to-right, (c) centerline-to-side and (d) side-to-centerline. We analyze them in depth in the main text, leading to our final decision to adopt (d) for more sensible representation.

the worm individual. In this subsection, we present how we represent the worm body coordinate for learning to regress.

\subsubsection{Worm Body Coordinate Representation}

We would like to define a canonical coordinate system for the worm body which we generically refer to as the UVcoordinate system (in contrast to the XY-coordinate system describing pixel coordinates in the image). A natural choice is to consider one coordinate that specifies the location along the anterior-posterior axis (from head to tail) and a second to be the dorsal-ventral axis (from back to belly) as worms typically "swim" on their sides as viewed through the microscope. Given these axes, there remains flexibility in exactly what coordinates to use and how they are scaled.

In this paper, we take U-coordinate as the the distance along the worm centerline (in pixels or percent-bodylength), from tail to head. For the V-coordinate we considered three different representations which are visualized in Figure 3 While it is generally straightforward to translate 
between these representations, in practice we found that the choice of representation effects learning and prediction accuracy.

Left-to-Right Representation as shown in Figure 3 (a) is the most straightforward approach. Similar to the Ucoordinate, we could take the V-coordinate to run left-toright orthogonal to the centerline and range from zero up to the width. However, we find that such a left-to-right Vcoordinate is a difficult regression target since the value is large on the right edge and then changes to 0 on the background. Such a sharp change can make learning struggle on the right, causing problematic artifacts during inference. Moreover, we note that in the lab environment, worms can roll over which makes identifying the true left (ventral) and right (dorsal) difficult, even for human annotators.

Centerline-to-side Representation is another we consider where the centerline has a fixed maximum coordinate value (e.g., the maximum observed width), and the V-coordinate decreases in proportion to the distance from the centerline. Figure 3 (b) shows a visualization this representation. It remedies the sharp change on the worm body edge and avoids dorsal/ventral ambiguity. However, such a representation tends to result in artifacts near the head and tail where the width goes to zero.

Side-to-Centerline Representation. We found the best V-coordinate representation is to utilize the distance from the side as depicted in Figure 3 (c). "Sides-to-Centerline" means that, instead of starting from centerline, the Vcoordinate indicates the distance to the (nearest) boundary orthogonal to the centerline. This is similar to a distance transform and has the appealing properties that the boundary has a U-value of 0 and the maximum value, located at the centerline indicates the width of the body at that Vcoordinate.

Straightening. As an alternative to visualizing the predicted UV-coordinates in the original image plane, we can "straighten" the worm by using the predicted coordinates to warp the worm image into a standardized coordinate system. Figure 2 shows an example of such a visualization where the bottom panel shows brightness values from the original image displayed in a canonical pose. When performing this mapping for the centerline-to-side representation, we disambiguate the V-coordinates for the left and right sides based on the predicted head-tail orientation.

\subsubsection{Worm Body Coordinate Regression}

Similar to worm segmentation module, we also turn to a U-Net architecture for dense regression at pixel level. The main task is to regress every pixel into UV coordinates. However, we note that the worm body is what we really care about, but not the background. Therefore, we also train this module to output a fine segmentation mask indicating where the UV-coordinates are valid.

For learning the segmentation mask output, we use the same binary cross entropy loss as Eq. 3.1 to train for segmentation, as denoted by $L_{\text {seg }}^{\text {fine }}$ (fine segmentation as opposed to coarse segmentation in the first module). For UVcoordinate regression, we simple adopt the L1 loss as below (we omit notation sum-over-images for brevity):

$$
\begin{aligned}
& L_{U}=\sum_{s=1, i \in \mathcal{I}_{s}}^{S} m_{i}^{s} \cdot\left|u_{i}^{s}-\bar{u}_{i}^{s}\right|, \\
& L_{V}=\sum_{s=1, i \in \mathcal{I}_{s}}^{S} m_{i}^{s} \cdot\left|v_{i}^{s}-\bar{v}_{i}^{s}\right|,
\end{aligned}
$$

where $m_{i}^{s}$ is output (after sigmoid transformation of the logits) from the current segmentation output $\mathbf{M}^{s}$ at scale $s$.

We note that an alternative would be to mask the loss using the ground-truth segmentation. Interestingly, we found that masking with the predicted mask yielded slightly better models. We conjecture the reason is that the predicted segmentation mask aligns better with the worm shape and allows the model to ignore some "hard" pixels that are included in the ground-truth segmentation mask.

In summary, the total loss to train the body coordinate regression module is the following:

$$
L_{r e g}=L_{U}+L_{V}+L_{\text {seg }}^{\text {fine }}
$$

\subsection{Worm Age Estimation}

With similar motivation as in worm body coordinate regression, we hypothesize that if a model is able to predict worm age, it must learn to leverage internal structure of the worm individual to some extent. Such learned knowledge will in turn help us investigate worm health status.

Worm age estimation is essentially a regression problem, regressing the input image into a continuous value indicating the predicted age (in hours). Therefore, we build upon a simple feed-forward ResNet34 network [13], modifying its last layer to output a single continuous value. The same backbone enables us to study how pre-training helps improve age estimation, either fine-tuning from ImageNetpretrained model, or the one trained for body coordinate regression. Similar to body coordinate regression, we simply adopt the L1 loss summing over all the $N$ images, the ground-truth age in minutes $a_{j}$ and the predicted age $a_{j}^{\prime}$ for a specific image $j$ :

$$
L_{\text {age }}=\sum_{j=1}^{N}\left|a_{j}-a_{j}^{\prime}\right| .
$$

We do not re-weight pixel-level loss w.r.t ground-truth ages. We find that the simple L1 loss suffices, introducing little biased prediction as demonstrated in experiments on the ground-truth and prediction distribution over ages. 


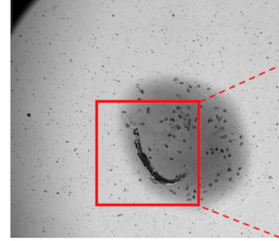

(a) Original Image
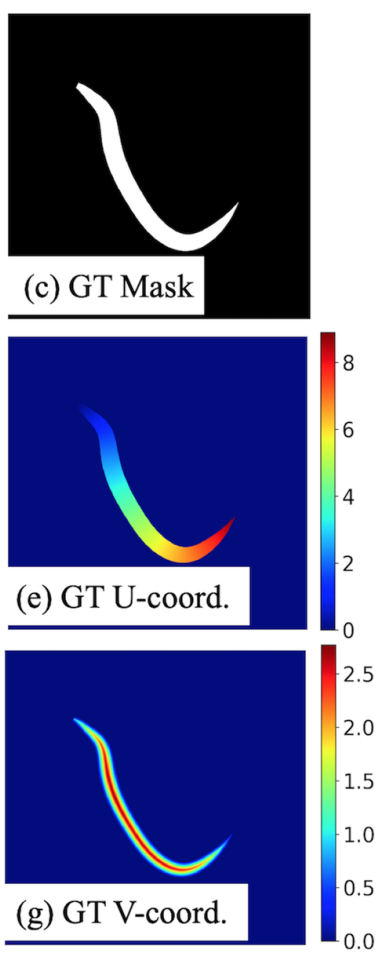

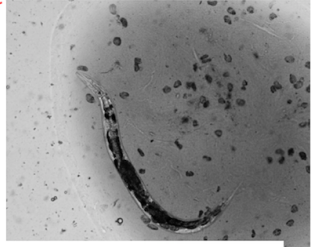

(b) Cropped Image
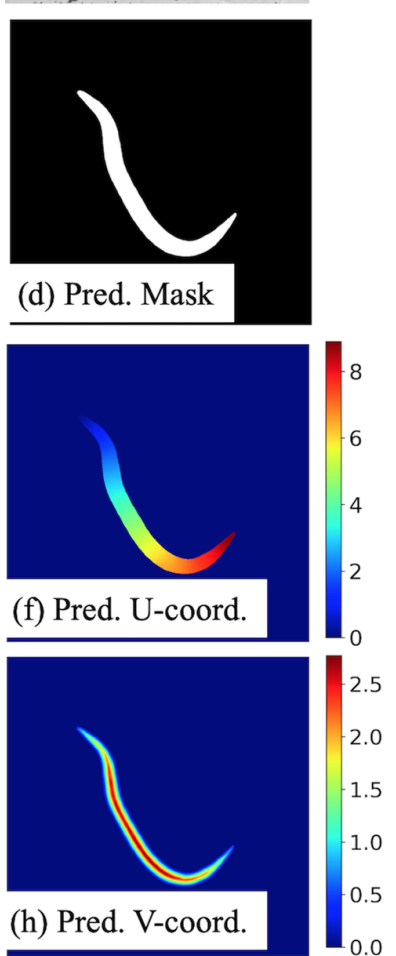

Figure 4: Worm body coordinate regression model takes as input the sub-image (b) which is cropped from the original image (a), and outputs worm segmentation mask (d) and UV coordinate predictions (f) and (h), respectively.

\section{Experiment}

We start by describing our dataset and our implementations, before diving into each model and validating performance of each.

\subsection{Implementation Details}

Dataset. Individual worms were imaged at $5 \mathrm{x}$ or $7 \mathrm{x}$ resolution at regular intervals $(\sim 3.5$ hours $)$ from larval stage to death, yielding a final dataset of 10,075 16-bit high resolution images, each of which contains a single worm. To annotate UV coordinates we leveraged the zlab toolbox ${ }^{1}$ which allows the user to draw the centerline as a spline curve and indicate other keypoints corresponding to organs

\footnotetext{
${ }^{1}$ https:/github.com/zplab/elegant
}

Table 1: Comparison of worm segmentation by the coarse segmentation module and the body coordinate regression module. We report Intersection-over-Union (IoU) and pixel classification accuracy (Acc.). Larger numbers mean better performance $\uparrow$.

\begin{tabular}{c|c|c}
\hline & Coarse Segm. & Fine Segm. \\
\hline IoU & 0.8482 & $\mathbf{0 . 9 0 3 3}$ \\
\hline Acc. & 0.9945 & $\mathbf{0 . 9 9 6 4}$ \\
\hline
\end{tabular}

and ground-truth segmentation mask. Annotation and curation was performed by a group experienced biologists

Over our annotated worm dataset, we split it into training (9,056 images) and validation (1,019 images). Our split was performed w.r.t worm identities so that a given individual worm never appears in both training and test.

Network Design. We use ResNet34 [13] as the encoder/backbone for all our three models. We observe that, given the amount of training data available, deeper models do not necessarily improve performance further. For perpixel prediction by worm segmentation and body coordinate regression, we build a decoder with skip connection to the encoder with same structure, consisting upsampling layers, convolution layers, ReLU layers [26] and Batch Normalization layers [14].

Training. We train each model individually for 100 epochs using the Adam optimizer [19], with initial learning rate 0.0005 and coefficients 0.9 and 0.999 for computing running averages of gradient and its square. We decrease learning rate by half every 20 epochs. For age estimation model, we fine-tune a pre-trained checkpoint (e.g., the body-coordinate regression model) with new decoder initialized randomly. We train our model using PyTorch [27] on a single NVIDIA TITAN X GPU.

\subsection{Worm Segmentation}

We evaluate the accuracy of the segmentation masks predicted by both the coarse (low-res) segmentation model and the fine (high-res) mask. Note that the segmentation masks from the two models have different resolution, due to both resizing and cropping. For fair comparison, we resize the coarse segmentation mask (sigmoid transformed map) back to the original image size (using bilinear interpolation), binarize the rescaled map, and crop it to match the high-res sub-image. Then we have two segmentation masks that have the same size and same region. We report the performance measure w.r.t two metrics, mean Intersection-overUnion (IoU) over all the testing images, and mean accuracy over all pixels of all testing images.

Table 1 lists the comparison, showing that the second model performs much better than the first one in terms of learning to segment worms. This is because of two rea- 

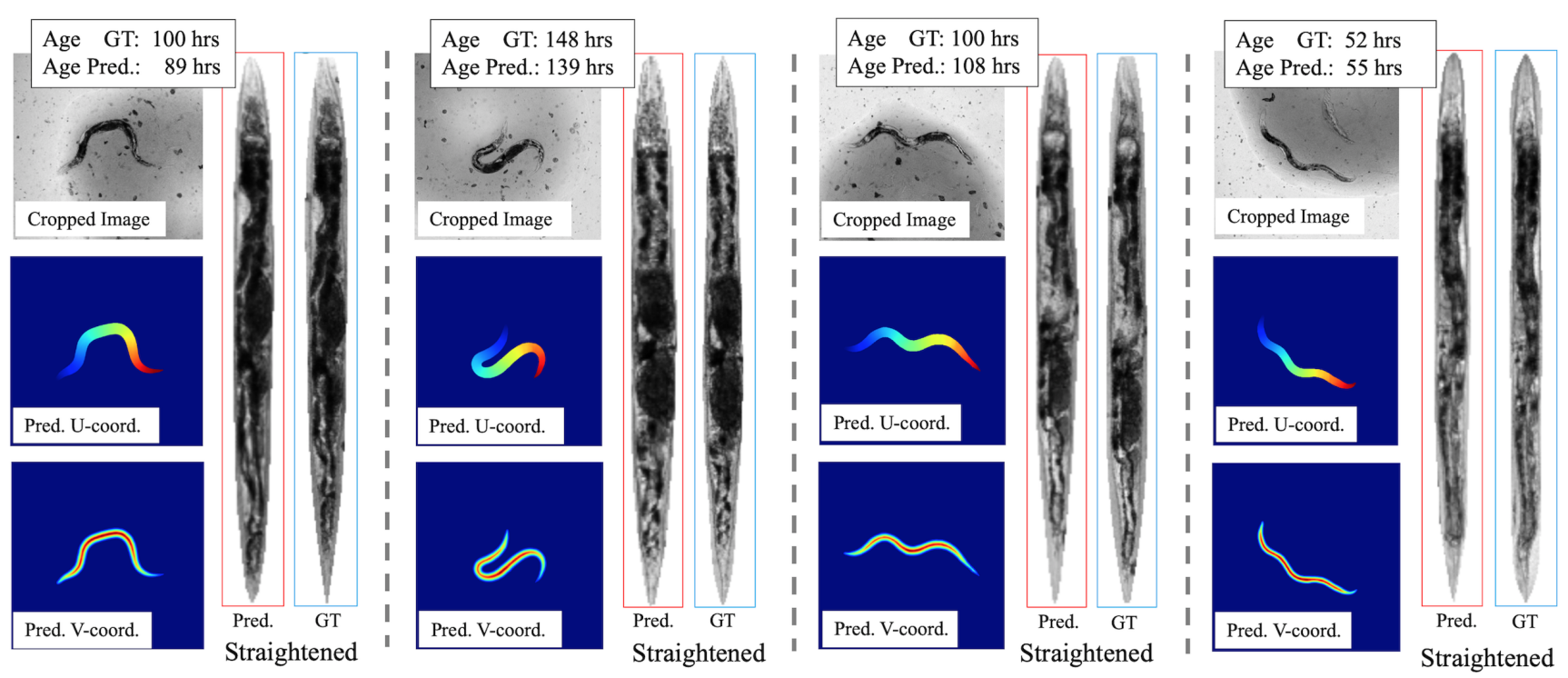

Figure 5: We visualize the body UV-coordinate regression output. With the ground-truth and predicted UV-coordinates, we straighten the worm into "canonical shape", respectively. Visually we can see the straightened worms match quite well between using predicted and ground-truth UV's.

Table 2: Average absolute error over for body coordinate regression, with or without masking in the loss function. We use the predicted segmentation mask to mask off background pixels. To compute Avg. Abs. Err., we average over both $\mathrm{U}$ and $\mathrm{V}$ predictions within the worm region specified by the ground-truth worm mask. Smaller error is better $\downarrow$.

\begin{tabular}{c|c|c}
\hline & w/ $/$ mask & w/ mask \\
\hline Avg. Abs. Err. & 0.3361 & $\mathbf{0 . 2 8 2 1}$ \\
\hline
\end{tabular}

sons. First, high-resolution input image as the input to the second model provides with finer-grained pixel information, which is helpful for predicting masks; whereas upsampling the coarse segmentation output may introduce artifacts that harm segmentation performance. Second, the first model works on smaller worms compared to the input image (biased towards the background), while the second model receives cropped image as input and thus works on a much balanced binary classification/segmentation problem. While the first model merely outputs a coarse segmentation mask, we note it is sufficient for us to crop the worm region based on the imperfect prediction, and move forward with fine segmentation and worm body regression. Figure 4(d) shows the segmentation mask by the second model.

\subsection{Worm Body Coordinate Regression}

For worm body coordinate regression, we measure the averaged L1 difference over all pixels between ground-truth UV coordinates and the predictions. However, as discussed, we have an option in training of whether to use the (predicted) segmentation mask to mask off background when computing the regression loss. Therefore, we compare two models that are trained without and with such a masking mechanism. Table 2 lists the comparison.

We can clearly see from Table 2 that with the masking mechanism during training, the model outperforms the counterpart (without masking) significantly. This demonstrates the benefit of enforcing the model to focus on the worm region, instead of all pixels of the input image. Figure 4 shows one example of predicted UV-coordinate and the fine segmentation mask, compared with the groundtruth; while Figure 5 shows more UV coordinate regression results.

It is worth noting that, with the predicted UV-coordinate, we can straighten the worms into a "canonical shape", allowing for better analysis w.r.t internal structures, e.g., organs' location and size. Figure 5 qualitatively compares the straightened worms by ground-truth UV and the predicted UV coordinates, respectively. We can see straightened worm are fairly comparable, enabling us to find specific organs to compare the size and location relative to the worm body.

\subsection{Worm Age Estimation}

We hypothesize that a model which is good at bodycoordinate regression will likely extract features of internal texture that are also useful for predicting age. We evaluate this using a pre-training transfer experiment. First we randomly initialize the weights to train from scratch. This 
Table 3: Age estimation performance measured by averaged absolute difference (over all validation images) with the groundtruth age (in hours). We compare three models (rows), training from scratch, fine-tuning from ImageNet-pretrained checkpoint and fine-tuning from our coordinate regression model. We also compare five different input format as listed in the five columns. Refer to the main text for detailed analysis.

\begin{tabular}{l|c|c|c|c|c}
\hline & Raw Image & Worm Only & Background (BG) & Silhouette & Silhouette+BG \\
\hline training-from-scratch & 18.50 & 31.08 & 36.03 & 69.23 & 18.51 \\
\hline ImageNet-pretrain & 17.63 & 17.94 & 33.35 & 65.89 & 18.06 \\
\hline coord.-reg.-pretrain & 14.50 & 20.90 & 26.43 & 65.08 & 17.00 \\
\hline
\end{tabular}

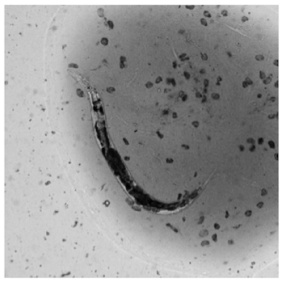

Cropped Image

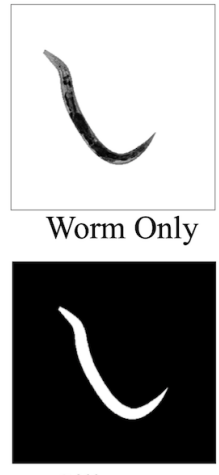

Silhouette

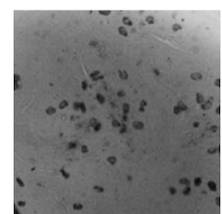

Background (BG)

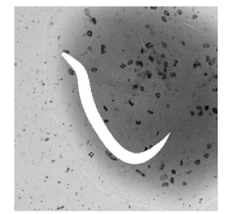

Silhouette + BG

Figure 6: Feature masking study in which we train models on different input modalities for worm age estimation. This helps disentangle which features are informative for ageestimation: the background environment, the worm size and shape (silhouette), or the internal structure of the worm individual. For example, even without looking at the worm it is possible to tell something about the age since the waste visible in the background increases over time.

provides a "lower bound" on the performance. Second, we follow a popular practice that we fine-tune from ImageNet pretrained checkpoint [9] for age estimation. Pretraining provides a better staring point than randomly initialized weights for targeted tasks. Third, we fine-tune our worm body coordinate regression model, which has already seen worm data and we expect such pre-training to perform better for age estimation.

Feature Masking A key challenge in drawing conclusions from powerful supervised machine learning models is that they easily latch on to image features which may be independent of the biology of interest but happen to be correlated. For predicting age, this could include factors such as the presence of tracks and waste in the growth media, condensation, drift in focus or illumination over time, etc. To control for these factors, we also evaluated how ageestimation accuracy varies when we mask aspects of the input image.

In addition to reporting the worm estimation performance with the input as the cropped Raw Image, we con-

sider the other four input formats, as shown in Figure 6 Worm Only means that we use the worm segmentation output to mask off all the background pixels, then we input this single worm to train for age estimation. This helps us understand how good age estimation we could achieve merely depending on features of the worm. Background (BG) is a random region cropped from the raw image but excluding any pixels belonging to the worm. We feed the background region to train for age estimation, with the real ground-truth age of the worm living in that environment. Note that background accumulates worm's waste, eggs and and tracks during time. Therefore the visual properties of the background are actually a good indicator of how long the worm has lived in the environment without seeing the animal itself. We consider an even more challenging setup, using silhouette and silouet te $+\mathrm{GB}$ to train for age estimation. The former helps show how much the worm shape and size correlate with its (estimated) age, while the latter will demonstrate how we can achieve with the combination of background information and shape.

Figure 7 plots age estimation error on the validation set with the models trained by fine-tuning the ImageNet pretrained checkpoint. We can see the models, even with different input formats, converge smoothly at the similar speed. Moreover, they do not overfit training data (or become worse on validation data), probably owing to the use of Batch Normalization in the models.

Table 3 lists detailed performance comparisons all the three models and five different input modalities. We note several interesting observations:

- Background indeed provides much information for age estimation, as we expected. The model trained on background only even outperforms the one trained on silhouette. This indicates the worm shape alone provides limited information for age estimation.

- Silhouette+BG shows much better performance than Background or Silhouette. This demonstrates the joint force by background and worm shape provides more informative cue for age estimation. However, we note that the predictive value of the background is entirely an artifact of the experimental condi- 
tion (starting with a clean environment) and thus likely has very little to do with the biology of aging itself ${ }^{2}$

- Using model pre-training outperforms substantially the model trained from scratch. Fine-tuning our coordinate regression model is even better than using the ImageNet-pretrained model with raw images as input, supporting our hypothesis that pretraining for body-coordinate regression results in useful features that capture the shape and content of the worm.

- Masking out the internal content of the worm (Silhouette+BG) decreases performance relative to the Raw Image, but the difference is small except for the coordinate-regression pretrained model which shows a much bigger benefit of adding the worm content (17.0 to 14.5 )

- While using raw image consistently gets the best predictive performance for each model, we note that Worm Only varies a lot for different models: ImageNet-pretrain works the best, outperforming coord.-re.-pretrain, whereas training-from-scratch does not show competitive results (which is understandable). We believe the reason is that, while coordinate regression model, as the pretrained checkpoint, has seen the worm images, it actually does not see Worm Only images which have very different distribution. However, the ImageNet-pretrained checkpoint offers a more generic feature extractor which makes itself more amendable for the Worm Only images through fine-tuning.

Age Distribution matching We plot the distribution of ground-truth and age predictions in Figure 8 (a) scatter plot to visualize the correlation between prediction and groundtruth, and (b) overlaid histograms to understand how the predictions match the ground-truth overall. We can see age prediction aligns quite well with the ground-truth. From the scatter plot we can see the smallest errors are for juvenile worms ( $<100$ hours) and middle aged (180-300 hours) with some decrease in accuracy near the end of life $(>300$ hours).

\section{Conclusion and Future Work}

We introduce "Celeganser", a prototyped automated system that assists analysis on single C. elegans worm in terms of studying lifespan and healthspan. Through extensive experiments, we validate the modules in Celeganser, including semantic segmentation, body coordinate regression and

\footnotetext{
${ }^{2}$ Of course the availability of food and other environmental features and stressors certainly do affect aging but in the context of the present experiment they are not controlled for independent of age and hence should be ignored.
}

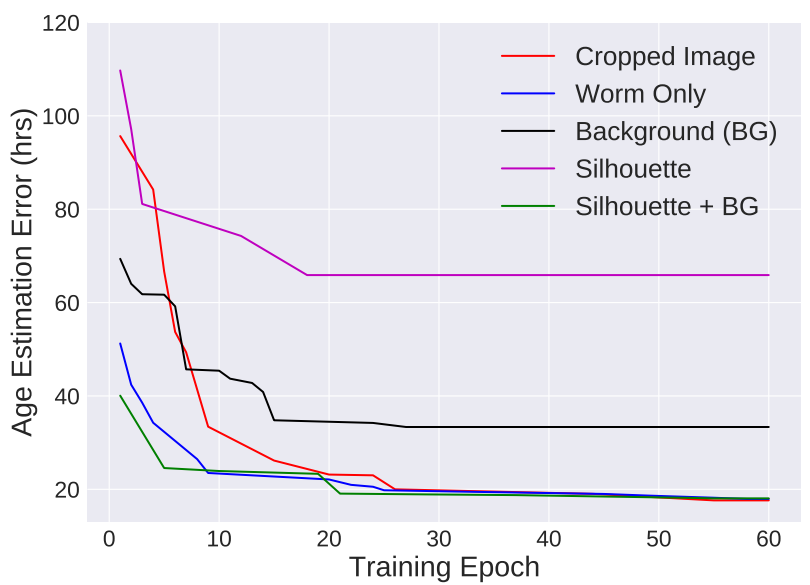

Figure 7: We plot the age estimation error in the validation set in the first 60 epochs. The models chosen here are the ones fine-tuned from an ImageNet-pretrained checkpoint, with different input formats. We can see all models converge at the similar speed, and slowly keep decreasing over time. Moreover, none of the models overfit (or get worse) even being trained long enough.
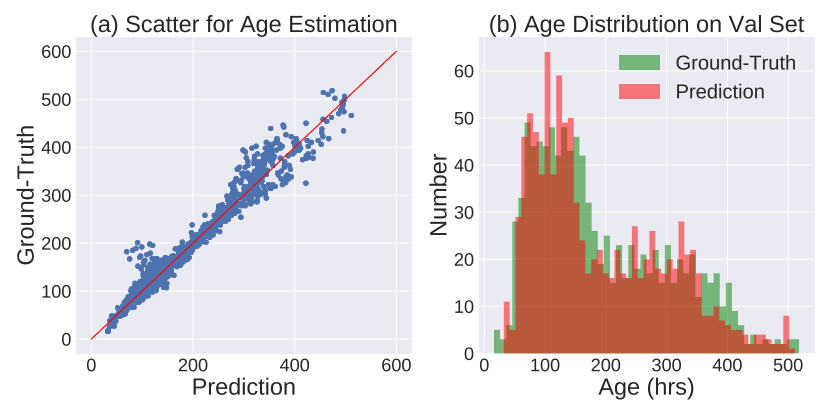

Figure 8: We demonstrate that the age prediction results align well with the ground-truth ages, based on two plots, (a) scatter plot of ground-truth vs. prediction of age estimation, and (b) histograms of ground-truth and prediction age in the validation set.

age estimation. The system already achieves high-levels of accuracy and deploying this system will allow for highthroughput analysis and provide interpretable and useful morphological features for studying life/health-span. We hope our study also sheds light on how to represent UVcoordinate and the importance of feature masking experiments in deriving biological insights from machine learning models.

\section{Acknowledgements}

The authors gratefully acknowledge Logan Tan, Nicolette Laird and Aditya Somisetty of the Pincus Lab who created and curated the ground-truth image annotations. This research was supported by NIH grant NIA R01AG057748, 
NSF grants IIS-1813785 and IIS-1618806, a research gift from Qualcomm, and a hardware donation from NVIDIA. Shu Kong also acknowledges Kleist Endowed Fellowship for the generous support of inter-disciplinary research.

\section{References}

[1] Rıza Alp Güler, Natalia Neverova, and Iasonas Kokkinos. Densepose: Dense human pose estimation in the wild. In Proceedings of the IEEE Conference on Computer Vision and Pattern Recognition, pages 7297-7306, 2018.

[2] Riza Alp Guler, George Trigeorgis, Epameinondas Antonakos, Patrick Snape, Stefanos Zafeiriou, and Iasonas Kokkinos. Densereg: Fully convolutional dense shape regression in-the-wild. In Proceedings of the IEEE Conference on Computer Vision and Pattern Recognition, pages 67996808, 2017.

[3] Simon Baeriswyl, Médéric Diard, Thomas Mosser, Magali Leroy, Xavier Manière, François Taddei, and Ivan Matic. Modulation of aging profiles in isogenic populations of caenorhabditis elegans by bacteria causing different extrinsic mortality rates. Biogerontology, 11(1):53, 2010.

[4] Sydney Brenner. The genetics of caenorhabditis elegans. Genetics, 77(1):71-94, 1974.

[5] Liang-Chieh Chen, George Papandreou, Iasonas Kokkinos, Kevin Murphy, and Alan L Yuille. Deeplab: Semantic image segmentation with deep convolutional nets, atrous convolution, and fully connected crfs. IEEE transactions on pattern analysis and machine intelligence, 40(4):834-848, 2017.

[6] Steven J Cook, Travis A Jarrell, Christopher A Brittin, Yi Wang, Adam E Bloniarz, Maksim A Yakovlev, Ken CQ Nguyen, Leo T-H Tang, Emily A Bayer, Janet S Duerr, et al. Whole-animal connectomes of both caenorhabditis elegans sexes. Nature, 571(7763):63-71, 2019.

[7] Nicolas Coudray, Paolo Santiago Ocampo, Theodore Sakellaropoulos, Navneet Narula, Matija Snuderl, David Fenyö, Andre L Moreira, Narges Razavian, and Aristotelis Tsirigos. Classification and mutation prediction from non-small cell lung cancer histopathology images using deep learning. $\mathrm{Na}$ ture medicine, 24(10):1559-1567, 2018

[8] James W Curtsinger, Hidenori H Fukui, David R Townsend, and James W Vaupel. Demography of genotypes: failure of the limited life-span paradigm in drosophila melanogaster. Science, 258(5081):461-463, 1992.

[9] Jia Deng, Wei Dong, Richard Socher, Li-Jia Li, Kai Li, and Li Fei-Fei. Imagenet: A large-scale hierarchical image database. In Proceedings of the IEEE Conference on Computer Vision and Pattern Recognition (CVPR), pages 248255. Ieee, 2009.

[10] David Eigen and Rob Fergus. Predicting depth, surface normals and semantic labels with a common multi-scale convolutional architecture. In Proceedings of the IEEE international conference on computer vision, pages 2650-2658, 2015

[11] Andre Esteva, Brett Kuprel, Roberto A Novoa, Justin Ko, Susan M Swetter, Helen M Blau, and Sebastian Thrun. Dermatologist-level classification of skin cancer with deep neural networks. Nature, 542(7639):115-118, 2017.
[12] Pierre Gönczy and Lesilee S Rose. Asymmetric cell division and axis formation in the embryo. WormBook, page 1, 2005.

[13] Kaiming He, Xiangyu Zhang, Shaoqing Ren, and Jian Sun. Deep residual learning for image recognition. In Proceedings of the IEEE Conference on Computer Vision and Pattern Recognition (CVPR), pages 770-778, 2016.

[14] Sergey Ioffe and Christian Szegedy. Batch normalization: Accelerating deep network training by reducing internal covariate shift. arXiv preprint arXiv:1502.03167, 2015.

[15] Thomas E Johnson, Deqing Wu, Patricia Tedesco, Shale Dames, and James W Vaupel. Age-specific demographic profiles of longevity mutants in caenorhabditis elegans show segmental effects. The Journals of Gerontology Series A: Biological Sciences and Medical Sciences, 56(8):B331-B339, 2001.

[16] Cynthia Kenyon, Jean Chang, Erin Gensch, Adam Rudner, and Ramon Tabtiang. A c. elegans mutant that lives twice as long as wild type. Nature, 366(6454):461-464, 1993.

[17] Cynthia J Kenyon. The genetics of ageing. Nature, 464(7288):504-512, 2010.

[18] Koutarou D Kimura, Heidi A Tissenbaum, Yanxia Liu, and Gary Ruvkun. daf-2, an insulin receptor-like gene that regulates longevity and diapause in caenorhabditis elegans. Science, 277(5328):942-946, 1997.

[19] Diederik P Kingma and Jimmy Ba. Adam: A method for stochastic optimization. arXiv preprint arXiv:1412.6980, 2014.

[20] Shu Kong and Charless C Fowlkes. Recurrent scene parsing with perspective understanding in the loop. In Proceedings of the IEEE Conference on Computer Vision and Pattern Recognition, pages 956-965, 2018.

[21] Alex Krizhevsky, Ilya Sutskever, and Geoffrey E Hinton. Imagenet classification with deep convolutional neural networks. In Advances in neural information processing systems, pages 1097-1105, 2012.

[22] Yann LeCun, Léon Bottou, Yoshua Bengio, and Patrick Haffner. Gradient-based learning applied to document recognition. Proceedings of the IEEE, 86(11):2278-2324, 1998.

[23] Jiunn-Liang Lin, Wei-Liang Kuo, Yi-Hao Huang, Tai-Lang Jong, Ao-Lin Hsu, and Wen-Hsing Hsu. Using convolutional neural networks to measure the physiological age of caenorhabditis elegans. IEEE/ACM Transactions on Computational Biology and Bioinformatics, 2020.

[24] Jonathan Long, Evan Shelhamer, and Trevor Darrell. Fully convolutional networks for semantic segmentation. In Proceedings of the IEEE conference on computer vision and pattern recognition, pages 3431-3440, 2015.

[25] William Mair, Patrick Goymer, Scott D Pletcher, and Linda Partridge. Demography of dietary restriction and death in drosophila. Science, 301(5640):1731-1733, 2003.

[26] Vinod Nair and Geoffrey E Hinton. Rectified linear units improve restricted boltzmann machines. In Proceedings of the 27th international conference on machine learning (ICML10), pages 807-814, 2010.

[27] Adam Paszke, Sam Gross, Soumith Chintala, Gregory Chanan, Edward Yang, Zachary DeVito, Zeming Lin, Alban Desmaison, Luca Antiga, and Adam Lerer. Automatic differentiation in pytorch. 2017. 
[28] Zachary Pincus. Ageing: A stretch in time. Nature, 530(7588):37-38, 2016.

[29] Zachary Pincus, Travis C Mazer, and Frank J Slack. Autofluorescence as a measure of senescence in c. elegans: look to red, not blue or green. Aging (Albany NY), 8(5):889, 2016.

[30] Olaf Ronneberger, Philipp Fischer, and Thomas Brox. Unet: Convolutional networks for biomedical image segmentation. In International Conference on Medical image computing and computer-assisted intervention, pages 234-241. Springer, 2015.

[31] Sayed Mohammad Ebrahim Sahraeian, Ruolin Liu, Bayo Lau, Karl Podesta, Marghoob Mohiyuddin, and Hugo YK Lam. Deep convolutional neural networks for accurate somatic mutation detection. Nature communications, 10(1):110, 2019.

[32] Nicholas Stroustrup, Bryne E Ulmschneider, Zachary M Nash, Isaac F López-Moyado, Javier Apfeld, and Walter Fontana. The caenorhabditis elegans lifespan machine. $\mathrm{Na}$ ture methods, 10(7):665, 2013.

[33] John E Sulston and H Robert Horvitz. Post-embryonic cell lineages of the nematode, caenorhabditis elegans. Developmental biology, 56(1):110-156, 1977.

[34] James W Vaupel, James R Carey, Kaare Christensen, Thomas E Johnson, Anatoli I Yashin, Niels V Holm, Ivan A Iachine, Väinö Kannisto, Aziz A Khazaeli, Pablo Liedo, et al. Biodemographic trajectories of longevity. Science, 280(5365):855-860, 1998. 